\title{
Modification of Kraft Wood-Pulp Fibre with Silica for Surface Functionalisation
}

Karen T. Love ${ }^{1}$, Brian K. Nicholson ${ }^{2}$, John Lloyd $^{1}$, Robert A. Franich ${ }^{3}$, R. Paul Kibblewhite ${ }^{1}$ and Shawn D. Mansfield ${ }^{4}$.

${ }^{1}$ Ensis Papro, Private Bag 3020, Rotorua, New Zealand

${ }^{2}$ Chemistry Department, The University of Waikato, Private Bag 3105, Hamilton, New Zealand

${ }^{3}$ Ensis Wood Processing, Private Bag 3020, Rotorua, New Zealand

${ }^{4}$ Department of Wood Science, University of British Columbia, 4030-2424 Main Mall, Vancouver, BC, Canada

\begin{abstract}
A new science strategy was devised in which glass surface properties would be imparted to woodderived fibre so that the enhancements known from addition of silane reagents to glass fibre-polymer composites could be realised for modified cellulose fibre-polymer composites. A process is described whereby the internal void spaces and micropores of never-dried Kraft pulp fibre walls were impregnated with silica, without substantially affecting the fibre integrity. This was achieved by initial dehydration of never-dried fibre through azeotropic distillation to achieve substitution of fibre water with the silicon chemical over a range of concentrations. Specific chemical reaction of the prepared fibre with the novel reagent $\mathrm{ClSi}(\mathrm{OEt})_{3}$ (which is more reactive than $\mathrm{Si}(\mathrm{OEt})_{4}$ ) was followed by base-catalysed hydrolysis of the ester groups to form a fibre-bound silica composite. As a result of this process, Kraft fibres were stiffened and made resistant to collapse. The physico-chemical substitution of water from micropores and internal voids of never-dried fibre with property-modifying chemicals offers possibilities in the development of new fibre characteristics, including fibres which may be hardened, plasticised, and/or stabilised against moisture, biodegradation or fire. The embedded silica may also be used as sites of attachment for coupling agents to modify the hydrophilic character of the fibre or to functionalise the fibre surface.
\end{abstract}

\section{Introduction}

Fibre derived from radiata pine wood using the Kraft pulping process has parameters, such as fibre length and wall cross-sectional area (Kibblewhite, 1989) which are ideal for its use in reinforcement of polymer composites (Woodhams et al., 1984) (Miller, 1994). Wood fibre-reinforced polymer composites tend to have low strength however, due to the incompatibility of the hydrophilic fibres and the hydrophobic polymer matrix. The fibre-polymer interface needs to be sufficiently strong for the fibres to effectively reinforce the composite. For wood fibre-thermoplastic composites, the molecular incompatibility of the hydrophilic cellulose fibre which can have adsorbed water (Beshay et al., 1985), 
and a hydrophobic polymer can result in reduction of composite strength with increase in volume fraction of the fibre, despite the fibre being the stronger component (Woodhams et al., 1984).

Surface incompatibility has also been encountered in the manufacture of glass fibre and plastic composite materials. Silane-based coupling reagents have been used to enhance the interfacial bonding between glass fibres and polymer matrices (Plueddemann, 1984). Coupling reagents generally are silicon compounds with functional groups which can react both with silanol surfaces on glass and with functional groups of polymers, or otherwise interact with polymers through mechanical or physical (e.g., van der Waal's forces) mechanisms. Silane coupling agents have been used to modify the hydrophilic character of wood fibres by bonding to the cellulose hydroxyls, forming C-O-Si silicate ester bonds (Raj et al., 1989) (Matuana et al., 1999) (Schneider and Brebner, 1985). Although application of silane coupling reagents for improvement of wood flour and wood-derived fibre polymer composites dates from 1983 (Xanthos, 1983), there is a paucity of fundamental knowledge of the reactivity of various coupling reagents with cellulose fibres and of the yield of coupled product. Chemical pre-treatment of fibre has been shown to have a modest influence on composite properties (Valadez-Gonzalez et al., 1999) (Pickering et al., 2003), but the gains fall far short of expectation according to the 'rule of mixtures' theory (Askeland, 1996) applied to the cellulose fibre-silane coupling reagent-polymer system.

One conclusion from these studies is that the C-O-Si covalent bond formed between cellulose and silicon is insufficiently stable to have any durable enhancement on wood fibre-polymer composite properties. A new science strategy was therefore devised in which glass surface properties would be imparted to wood-derived fibre so that the enhancements known from addition of silane reagents to glass fibre-polymer composites could be realised for modified cellulose fibre-polymer composites. By embedding silica within the fibre cell wall and attaching a silane coupling agent to silanol groups, stable $\mathrm{Si}-\mathrm{O}-\mathrm{Si}$ bonds would be produced. These bonds are more hydrolytically stable than the cellulose equivalent $\mathrm{C}-\mathrm{O}-\mathrm{Si}$ bonds, producing a fibre that should be more suitable for polymer composite use.

Silica modification of wood involves the incorporation of silica into the structure of wood or pulp fibre, and has been used to enhance the properties of wood as a building material (Saka et al., 1992) (Tshabalala and Gangstad, 2003) (Saka and Ueno, 1997). Silica modification of wood has also been used to produce mesoporous silica by sol-gel mineralization using the wood structure as a template (Shin et al., 2003) (Shin et al., 2005) (Qian et al., 2004) (Persson et al., 2004). The embedded silica may also be used as sites of attachment for coupling agents to modify the hydrophilic character of the fibre or to functionalise the fibre surface.

This paper reports the modification of Kraft pulp fibre by incorporating polymeric silica in the fibre wall. A subsequent paper will report on the development of a functionalised fibre surface using aminopropyltriethoxysilane coupling reagent covalently-bonded to the embedded silica. 


\section{Experimental}

Reagent-grade chemicals were used except where noted, and all chemicals were used as received without further purification.

\subsection{Pulp Fibre}

Radiata pine Kraft pulp was used in the modification experiments, with kappa numbers ranging from $<1$ to 110 . Slabwood chips and bleached Kraft pulp (kappa number $<1$, bleaching process $\mathrm{OODE}_{\mathrm{OP}} \mathrm{D}$ ) were kindly provided by Carter Holt Harvey Kinleith Mill, Tokoroa, New Zealand.

\subsection{Alkoxysilanes}

Silicon reagents used were tetraethoxysilane (TEOS, Aldrich, 98\%) and 'Silbond 40' (Ethyl Silicate 40, Silbond Corporation). Triethoxychlorosilane was synthesised from $\mathrm{SiCl}_{4}$ and $\mathrm{TEOS}$ according to the method of Chernyshev et al. (Chernyshev et al., 1995), which gave a mixture containing $\mathrm{Si}(\mathrm{OEt})_{4}$, $\mathrm{ClSi}(\mathrm{OEt})_{3}$ and $\mathrm{Cl}_{2} \mathrm{Si}(\mathrm{OEt})_{2}$ in approximate ratio 25:65:10 from the intensities of the ${ }^{29} \mathrm{Si}$ NMR signals at $\delta-82,-70$ and $-56 \mathrm{ppm}$ respectively. This mixture, hereafter referred to as TECS, was used for the experiments being described in this report.

\subsection{Typical Silica Modification Procedure (Using Tetraethoxysilane or 'Silbond 40’)}

Azeotropic distillation was carried out using a Dean-Stark water separation trap and distillation apparatus. The never-dried un-bleached Kraft fibre (12.6 g or $25 \mathrm{~g}$ oven-dried wt, kappa no. 41-62) was immersed in a round-bottomed flask in the azeotropic solvent (toluene or ethyl acetate, $60 \mathrm{~mL}$ ) alone or with the silane (tetraethoxysilane, or 'Silbond 40') at concentrations of 1, 3, 10 and 30\% w/v. The fibres were kept immersed by a wire mesh and gently heated under reflux. When the collection of water had ceased, heating was continued for a further $30 \mathrm{~min}$. The fibre was then separated by decantation, allowed to cool and collected and drained in a Buchner funnel. The fibre was added to a dilute ammonia solution (1:10, 880-ammonia:water, $150 \mathrm{~mL})$ to initiate base-catalysed hydrolysis, and shaken at $250 \mathrm{rpm}$ overnight. Following this, the ammonia solution-soaked fibre was drained in a fine-mesh sieve, and washed with deionised water until a pH of 7-7.5 was reached, and then allowed to air-dry. Solvent-dried fibre was used as a control, as was the wet, untreated Kraft fibre. 


\subsection{Optimised Silica Modification Procedure (Using Triethoxychlorosilane)}

Azeotropic distillation was carried out using a Dean-Stark water separation trap and distillation apparatus. The never-dried un-bleached Kraft fibre (12.6 g or $25 \mathrm{~g}$ oven-dried wt, kappa no. 41) was immersed in a round-bottomed flask in ethyl acetate $(60 \mathrm{~mL})$. The fibres were gently heated under reflux. When the collection of water had ceased, heating was continued for a further 30 min before the addition of triethoxychlorosilane mixture $(18 \mathrm{~mL})$. The pulp mixture was kept heated under reflux for a further 30 minutes. The fibre was then allowed to cool, separated by decantation, and collected and drained in a Buchner funnel and washed with ethyl acetate (approximately $100 \mathrm{~mL}$ ). The fibre was added to a dilute ammonia solution (1:10, 880-ammonia:water, $150 \mathrm{~mL})$, and shaken at $250 \mathrm{rpm}$ overnight. Following this, the ammonia solution-soaked fibre was drained in a fine-mesh sieve, and washed with deionised water until a $\mathrm{pH}$ of 7-7.5 was reached, and then allowed to air-dry.

\subsection{Determination of Silica Content of Modified Fibre}

The silica content within the fibre was determined by ashing the silica-fibre product at $520^{\circ} \mathrm{C}$ and weighing the residue. The residual weight of ashed un-modified fibre was used as the blank.

\subsection{Enzymatic Treatments of Silica-Modified Fibre}

Novozyme SP342 (Novo Nordisk, Bagsværd, Denmark) was used for cellulase treatments. Its activity was determined on carboxymethylcellulose (1\% CMC, Sigma) using the method described previously (Wood and Bhat, 1988). Both control and silica-modified pulp fibres were subject to cellulase treatments at $\sim 11 \mathrm{IU} /$ gram oven-dried fibre (3\% consistency in $50 \mathrm{mM}$ phosphate buffer, $\mathrm{pH} 7.0$ ) for 1 and 4 hours at $50^{\circ} \mathrm{C}$. All reactions were terminated by boiling for 15 minutes. Control pulps were similarly treated with equivalent quantities of heat inactivated enzyme (15 minutes boiling).

\section{$2.7{ }^{13} \mathrm{C}$ and ${ }^{29} \mathrm{Si}$ NMR Spectroscopy}

Silica-modified pulp samples were milled in a Wiley mill, packed into a $7 \mathrm{~mm}$ zirconia rotor, and spun at $5 \mathrm{kHz}$. Solid state ${ }^{29} \mathrm{Si}$ NMR spectra were obtained from a Bruker Avance 200 NMR spectrometer, using an acquisition time of $0.2 \mathrm{~s}$ and recycle delay of $2.0 \mathrm{~s} .{ }^{29} \mathrm{Si}$ spectra were externally referenced to acid-washed sand, which was ground to a fine powder in a ring mill. Solution state ${ }^{13} \mathrm{C}$ and ${ }^{29} \mathrm{Si} \mathrm{NMR}$ spectra were obtained from a Bruker Avance 300 NMR spectrometer using standard acquisition methods. 
$\mathrm{CDCl}_{3}$ was used as the solvent lock and chemical shifts were internally referenced to tetramethylsilane $\delta 0 \mathrm{ppm}$.

\subsection{Fibre Cross-Sectional Dimensions Analysis}

Cross-section fibre dimensions of thickness, width, wall area and wall thickness were measured using image processing procedures described in detail elsewhere (Kibblewhite and Bailey, 1988). The parameters of width, thickness and wall area were measured for dried fibres re-wetted from handsheets prepared from the fibre materials. The product of fibre width $\times$ fibre thickness represents the minimum fibre cross-section rectangle. The ratio width:thickness is an indicator of the collapse potential of the dried and re-wetted fibres. The greater the width and the lower the thickness of a fibre cross-section, the greater is the extent of fibre collapse.

\subsection{Scanning Electron Microscopy (SEM) and Energy-Dispersive Spectrometry (EDS)}

Fibre samples were dehydrated in ethanol and embedded in L.R. White acrylic resin. The resin blocks were trimmed on a microtome and mounted on SEM stubs using silver glue and coated with chromium.

SEM images were obtained using a JEOL 6700 field emission scanning electron microscope operated at an accelerating voltage of $10 \mathrm{kV}$. Samples were examined with a backscattered electron detector providing atomic number contrast to detect the location of silicon in relation to the fibre. The same field of view was then scanned using an energy dispersive X-ray spectrometer to acquire a set of X-ray maps for $\mathrm{Si}, \mathrm{C}$, and $\mathrm{O}$. Point acquisition rate of $1 \mathrm{~ms}$ was used for an array of $512 \times 384$ points for approximately 2 hours. X-ray spectra based on point or field of view scans were also acquired for comparison.

\subsection{Fourier Transform Infra-Red (FTIR) Spectroscopy}

FTIR spectra were obtained with a Bruker Vector 33 spectrometer. Samples were scanned using diffuse reflectance mode, in the DRIFT sample accessory, from 4000 to $500 \mathrm{~cm}^{-1}$. Fibre samples were ground in a Wiley mill prior to analysis.

\subsection{Transmission Electron Microscopy}


Fibre samples were dehydrated in acetone and embedded in Spurr's low viscosity resin. Ultrathin sections were cut with a diamond knife on an ultramicrotome, stained with $1 \%$ potassium permanganate (in 1\% citrate buffer) and then examined with a Philips 300 transmission electron microscope.

\section{Results and Discussion}

\subsection{Silica-Modification of Kraft Pulp Fibre using TEOS and 'Silbond 40'}

Silica-modification of Kraft pulp was facilitated by dehydrating never-dried fibre samples using a solvent which forms an azeotrope with water. The water in the fibres was removed concomitantly with substitution with the solvent, thereby mitigating distortion and collapse of the fibre structure which can be an artefact of other drying methods. In the case of fibre derived by a chemical wood pulping process, such as Kraft, water removal by water evaporation also causes pores in the fibre structure to collapse, and wall lamellae to adhere to one another. The walls of unbleached Kraft fibres are highly porous (Stone and Scallan, 1968) and are densified during pulp drying through structural elements within fibre walls being pulled together and bonded one to another (Nazhad and Paszner, 1994). The removal of water from wood pulp fibres can be viewed as a two-stage process, viz, the removal of water from pores between lamellae resulting in their lamination, and the dehydration of the microfibril matrix, resulting in the shrinkage of fibre walls (Weise and Paulapuro, 1995).

The addition of an alkoxysilane to the azeotropically-dried, solvent-saturated pulp sample allowed the silane to diffuse throughout the fibre cross-section. The silane-modified fibre could then be added to dilute ammonia solution, initiating base-catalysed hydrolysis of the alkoxysilane within the fibre to form initially silicic acid (Eqn 1), which would subsequently dehydrate and form polymeric silica within the fibre wall.

$$
\mathrm{Si}(\mathrm{OEt})_{4} \underset{\mathrm{H}_{2} \mathrm{O}}{\stackrel{\mathrm{NH}_{3}}{\longrightarrow}} \mathrm{Si}(\mathrm{OH})_{4}+4 \mathrm{EtOH} \quad \text { Equation } 1
$$

Initial $\mathrm{SiO}_{2}$ fibre modification experiments were carried out with TEOS. It was found that the incorporation of silica in the fibre was critically dependent on the extent of washing the treated fibre with the azeotrope solvent prior to hydrolysis. This was considered an important step to circumvent the precipitation of 'loose' silica with the modified fibre which could skew the ash content of the product. With minimal washing high $\mathrm{SiO}_{2}$ contents were achieved but these included deposits on the cell walls and within the lumen. On the other hand, thorough washing led to low levels of silica incorporation. These results indicated that TEOS was only physically absorbed into the fibre with no specific chemical reaction 
until the base was added. This made it difficult to achieve a consistent yield and uniform distribution of silica within the fibre wall only.

An alternative source of silica to TEOS is 'Silbond 40', which is a partially hydrolysed TEOS material, with an average of five silicon atoms per oligomer. Although the size of the TEOS molecule was expected to facilitate its diffusion through the fibre sample during the azeotropic drying process, Silbond, being a more bulky molecule, was considered less likely to be leached during the treated fibre washing steps. Donath et al. (Donath et al., 2004) has reported that although a higher deposition of silica within the cell wall was achieved when using monomeric silanes to modify solid wood, pre-hydrolysed silanes such as 'Silbond 40' gave a greater weight increase to the wood samples. This was found to be true in the present study.

In one example using 'Silbond 40' dissolved in toluene and never-dried Kraft fibre (kappa number 27), the ash content in the modified fibres was directly related to the concentration of the tetra ethyl silicate dissolved in the toluene during the azeotropic distillation process (Table i). However, microscopy examination of the product indicated that most of the silica produced appeared to be located in the fibre lumen. The filling of the fibre lumen has been shown to improve mechanical properties of the fibre such as hardness (Mai and Militz, 2004), which may be of benefit in the production of silica-modified wood fibre-polymer composites. However, the deposition of silica on the surface of the outer fibre walls and inner lumen walls is of little benefit for attachment of a silane coupling reagent as this prevents the uniform attachment of coupling agent residues on the surfaces of the fibre (Miyafuji and Saka, 1999).

Table i: Ash content of 'Silbond 40' modified Kraft fibre.

\begin{tabular}{ll}
\hline Fibre modification conditions & Ash content \% w/w \\
Untreated & 0.28 \\
Toluene only & $<0.15$ \\
1\%TES, 1:10 & 1.61 \\
3\%TES, 1:10 & 3.32 \\
10\%TES, 1:10 & 8.20 \\
30\%TES, 1:10 & 28.1 \\
30\%TES & 33.0 \\
30\%TES, 1:1 & 13.9 \\
30\%TES, 1:3 & 22.0 \\
30\%TES, 1:10 & 26.3 \\
30\%TES, 1:30 & 24.7 \\
\hline TES = Tetra-ethyl silicate as 'Silbond 40' \\
1:× ratio = 880 Ammonia:water ratio for silane hydrolysis.
\end{tabular}


Where silica had been incorporated into the fibre wall, this could be visualised using SEM-EDS analysis. An example of this is shown in Figure i, where the location and density of silicon as silica embedded in the fibre wall is shown in orange false-colour.
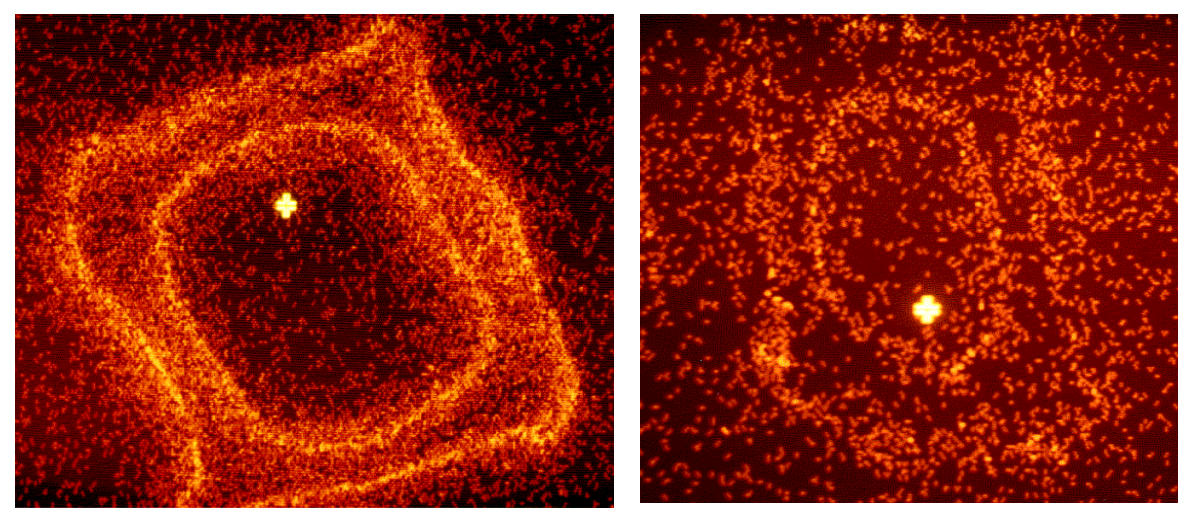

Figure i. SEM-EDS image of cross-section of Kraft fibre modified using toluene azeotrope containing Silibond 40 at 30\% w/v (left) and 10\% w/v (right) concentration and hydrolysed using aqueous ammonia.

Where silica filled the fibre lumen, this was also visualised using SEM-EDS, as shown in Figure ii, in this case using green false colour to highlight location of silicon. Some silicon is also located on the outer fibre wall, but the bulk is deposited in the lumen, adding significantly to the fibre density.

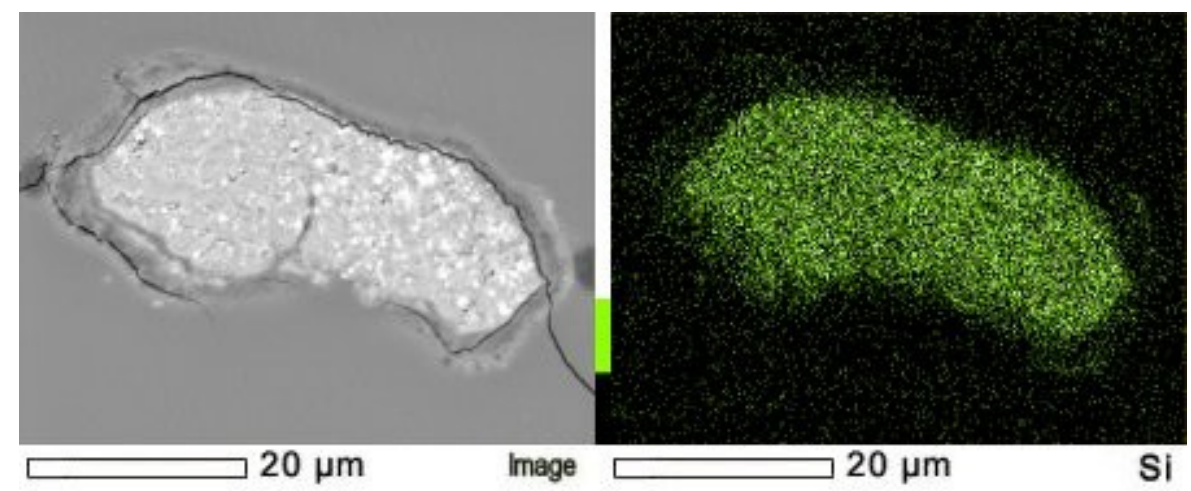

Figure ii: SEM (left) and silicon EDS image (right) of 'Silbond 40'-modified fibre showing lumen filled with silica.

The effect of the azeotrope drying process and silica modification on the never-dried pulp fibre dimensions are summarised in Table ii. The data show that, on natural drying, the water-swollen fibre undergoes shrinkage as expected (e.g., fibre wall area reduced from $349 \mu \mathrm{m}^{2}$ wet to $252 \mu \mathrm{m}^{2}$ dry). Silica modification had a significant effect increasing the fibre wall area with most of the samples prepared. It was also observed that toluene azeotrope drying alone produced a larger fibre than that obtained from 
passive air drying, and that qualitatively, this fibre was also appeared to be stiffer and more resistant to collapse than the air-dried fibre.

Table ii. Effect of toluene azeotrope drying and 'Silbond 40' silica modification of Kraft fibre dimensions.

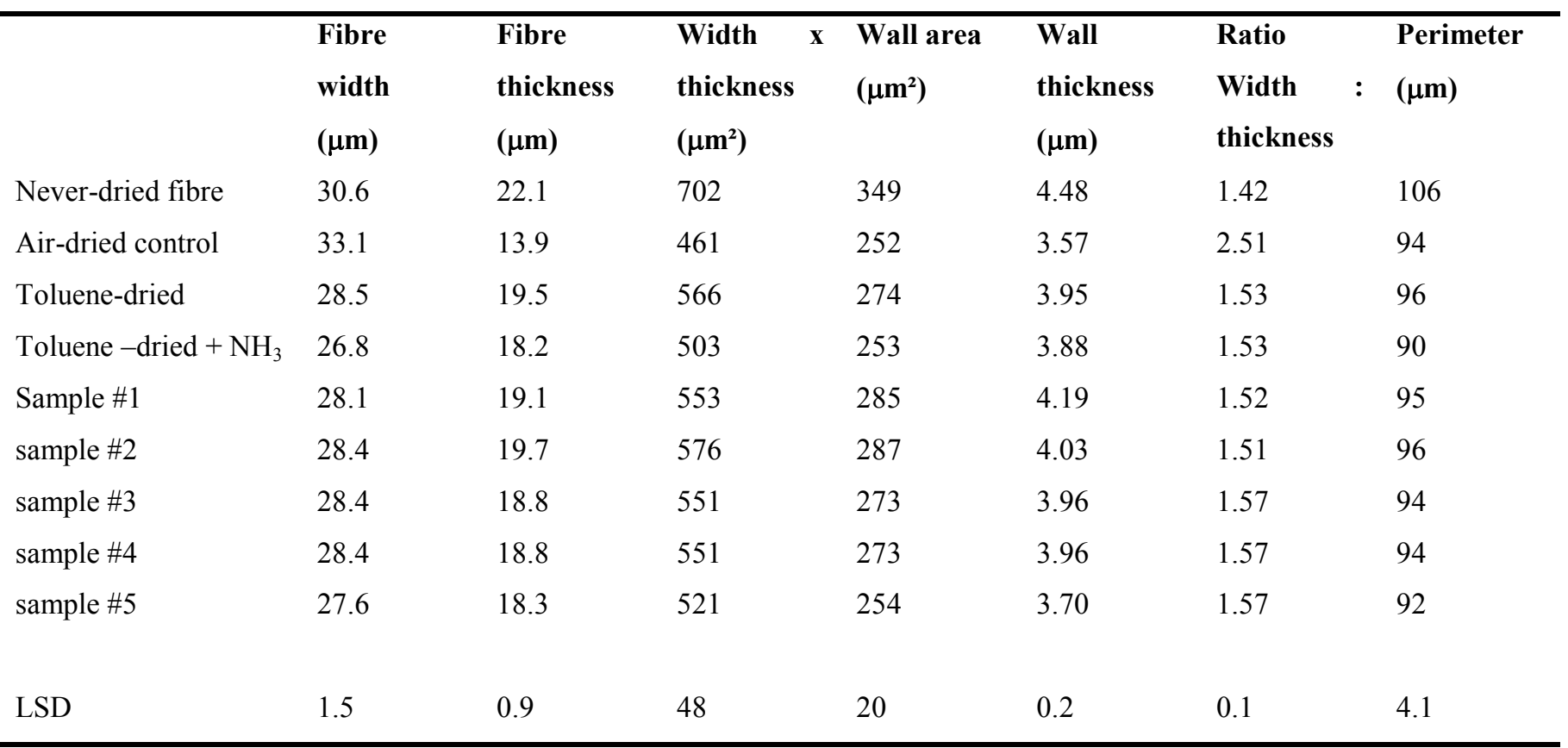

The silica-modified fibre material also appeared to be very tough, being resistant to ring-mill grinding for spectroscopy preparation. The modified fibre was also silky or slippery to the touch, suggesting that the silica polymer was accessible at the surface. This was confirmed using TEM (Figure iii) which shows the outer cross-section of the fibre wall with dark areas (silicon) concentrated at the surface, but also spasmodically within the fibre wall. The concentration of silica polymer at the fibre surface could account for the tactile and toughness properties. 

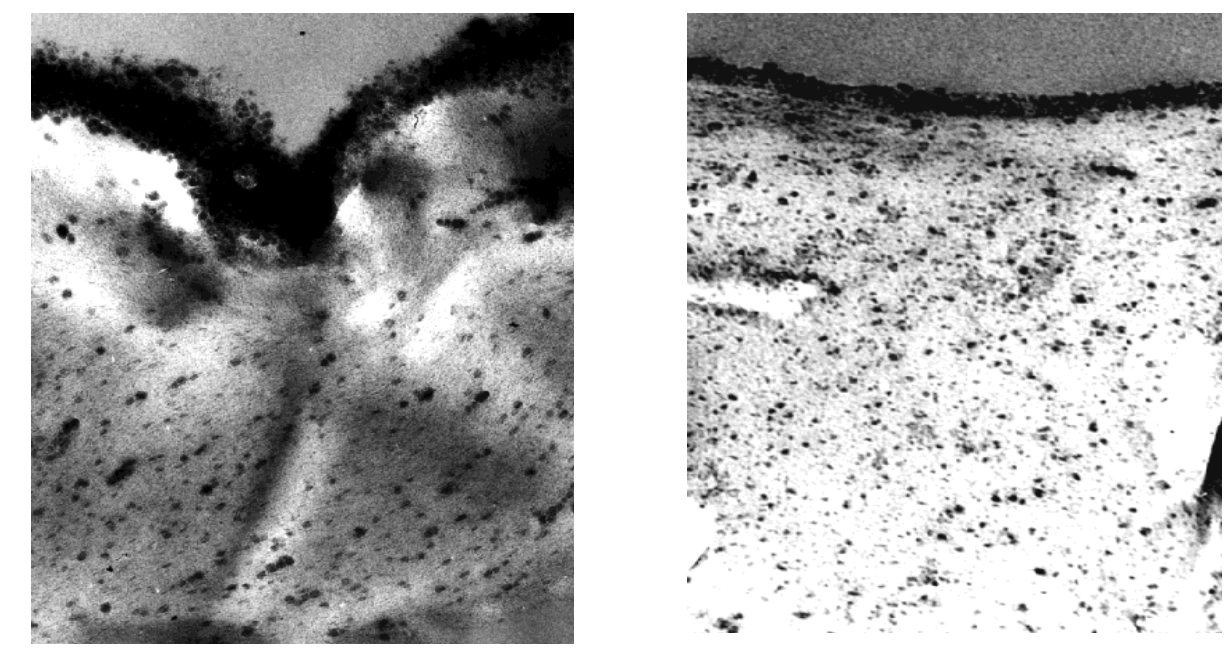

Figure iii. TEM image of silica-modified Kraft fibre using 'Silbond 40' 30\% w/v, (left) and 'Silbond $400^{\prime} 10 \% \mathrm{w} / \mathrm{v}$ (right). The dark regions locate presence of silicon.

Having established that the silica polymer was located most densely at the fibre surface using the 'Silbond 40' modification process, it was considered important to establish how well the silica polymer was embedded into the fibre wall. This was approached through use of cellulase enzyme treatment to dissolve, at least in part, the accessible cellulose. The data for this experiment are summarised in Table iii, which show that despite significant liberation of glucose during the hydrolysis, the effect on ash content of the residual fibre was insignificant. This implied that the silica was bound tightly into the fibre wall, but in addition, that the silica-modified surface of the fibre also partly impeded the accessibility of the enzyme to cellulose.

Table iii. Hydrolysis of cellulose in silica-modified Kraft pulp fibre

\begin{tabular}{lllll}
$\begin{array}{l}\text { 'Silbond 40' } \\
\text { treatment (\%) }\end{array}$ & $\begin{array}{l}\text { Treatulase } \\
\text { Dosage (IU/g) }\end{array}$ & $\begin{array}{l}\text { Time (min) } \\
\text { Glucose liberation }\end{array}$ & $\begin{array}{l}\text { Residual fibre ash } \\
\text { (mg/g) }\end{array}$ \\
\hline 0 & & & \\
\hline 0 & 0 & 240 & 0.10 & 0.87 \\
0 & 11 & 60 & 16.73 & 0.61 \\
10 & 11 & 240 & 23.69 & 0.55 \\
10 & 0 & 240 & 0.11 & 5.3 \\
10 & 11 & 60 & 15.51 & 5.9 \\
30 & 11 & 240 & 20.98 & 4.5 \\
30 & 0 & 240 & 0.16 & 20.7 \\
30 & 11 & 60 & 9.52 & 19.4 \\
& 11 & 240 & 16.01 & 19.5
\end{tabular}


Although use of toluene for the azeotropic distillation of water and silane modification was initially the preferred solvent based on the azeotrope distillation temperature and composition ( $20 \%$ water) for efficient fibre drying, ethyl acetate was found to be consistently preferable in terms of effectiveness for silane ingress and retention in the fibre based on silica content after ammonia-catalysed hydrolysis, despite having a lower water content of the azeotrope (8\%) which resulted in a slower drying rate. In one example, never-dried fibre (kappa number 41) was treated with both TEOS and 'Silbond 40' to give 3\% and $4 \%$ ash content respectively for the toluene azeotrope, while use of ethyl acetate gave ash content of $8 \%$ and $15 \%$ respectively. Use of ethyl acetate as the azeotrope solvent also had the benefit of a lower azeotrope temperature $\left(70^{\circ} \mathrm{C}\right.$, vs. $85^{\circ} \mathrm{C}$ for toluene) to expose the natural fibre to during the modification process.

The porosity of the Kraft pulp fibre, expressed as the kappa number, also had an effect on retention of silica in the fibre wall after treatment with either TEOS or 'Silbond 40' (Figure iv). Highly-porous fibre (kappa number $\leq 1$ ) after processing had less than 5\% ash, indicating that either the silane was readily extracted from the fibre after the azeotrope drying and treatment step, or the silica, as nano-sized particles formed in the initial polymerisation stages, was too small to be effectively trapped in the fibre structure and pores. For TEOS modification, the optimal fibre kappa number was 40 , giving $15 \%$ ash value. For 'Silbond 40', the optimal fibre kappa number was 60 , giving nearly $25 \%$ ash value. Modification of fibre having kappa number greater than 100 resulted in less than $15 \%$ ash, indicating that fibre of low porosity limited the ingress of the silane. A study by Kibblewhite and Bawden (Kibblewhite and Bawden, 1989) on unbleached Kraft pulp ranging in kappa from 38 to 117 revealed that with a decrease in kappa number, the width and thickness of the fibre also decrease, along with a decrease in the fibre cell wall, and in the lumen fraction of the fibre cross sectional area. The physical structure of the fibres at this kappa number range therefore may be more suited to impregnation by the various sized silanes.

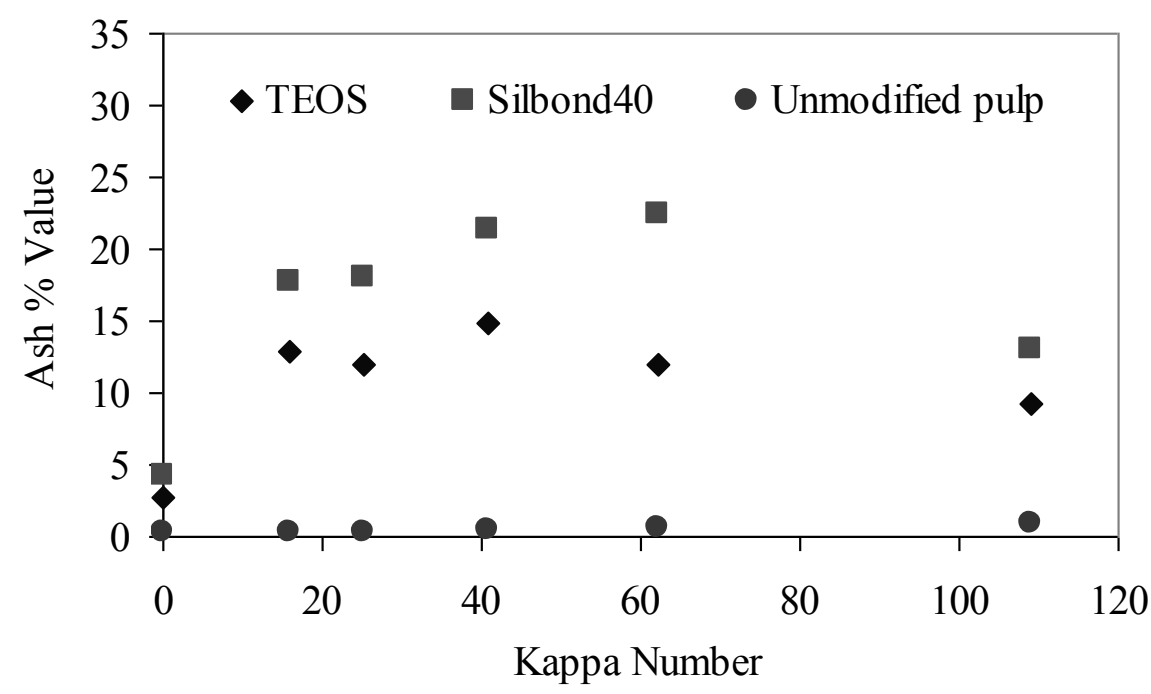


Figure iv. Silica content of Kraft pulp of different kappa number.

In summary, never-dried Kraft wood pulp fibre of medium porosity (range 40-60 kappa number) could be modified with the simple, monomeric silane, TEOS, and the oligomeric silane 'Silbond 40', to give, after ammonia-catalysed hydrolysis, fibre with silica polymer embedded within the wall, with the highest silica concentration at the fibre surface. There was, however, with the process described above, insufficient control over the destination of the silica, either in the fibre wall or in the fibre lumen or a combination of both when using TEOS or 'Silbond 40' and azeotropic water removal and exchange of the silicon compounds. Silica polymer embedded at the fibre wall surface had value for further modification using glass fibre silane coupling agents, whereas silica embedded in the lumen added less important physical properties, such as increased stiffness but at the expense of increased density, and no value for further surface modification. This conclusion led to a different strategy to use a silane modification reagent which would have better fibre wall retention through chemical reaction with the cellulose or lignin components prior to the hydrolysis step to give a fibre-wall bound silica polymer.

\subsection{Optimised Silica Modification Using Triethoxychlorosilane, TECS}

$\mathrm{Si}-\mathrm{Cl}$ bonds are more reactive towards substitution reactions than are $\mathrm{Si}-\mathrm{OEt}$ bonds, so $\mathrm{ClSi}(\mathrm{OEt})_{3}$ in ethyl acetate was explored to see if this could be used to chemically bond silicon to the fibre wall components, cellulose and lignin, via $\mathrm{C}-\mathrm{OH}$ groups, prior to more extensive hydrolysis to form silica polymer. This reagent does not appear to have been used before as a source of silica for wood or natural fibre modification.

TECS gave excellent results, with reproducible levels of silica incorporation in the fibre (up to $20 \%$ with fibre having kappa number in the range 16 to 41) and with less variance as a result of solvent washing procedures to remove any excess of reagent after the chemical reaction. Most importantly for subsequent addition of a silane coupling reagent, it was found, because of the chemical reactivity of the $\mathrm{Si}-\mathrm{Cl}$ group, that $\mathrm{ClSi}(\mathrm{OEt})_{3}$ was remarkably specific for incorporating silica within the fibre walls with little silica being deposited on the fibre surfaces or within the lumen.

The hydrochloric acid liberated during the reaction of TECS with hydroxyl groups was neutralised by addition of the fibre into excess ammonia solution. It is possible that the hydrochloric acid released during initial reaction of TECS with the fibre walls could initiate proton-catalysed cleavage of Si-OEt bonds leading to some degree of condensation to $\mathrm{Si}-\mathrm{O}-\mathrm{Si}$ bonds prior to the subsequent base-catalysed hydrolysis, and so contribute to the overall specificity of silica deposition in fibre walls. Hydrochloric acid can degrade the cellulose in the pulp fibre wall by the acidic hydrolysis of the glycosidic bonds (Sjostrom, 1981) but the presence of glucose was not analysed for in this study. 
Silica-modified fibre produced using the $\mathrm{ClSi}(\mathrm{OEt})_{3}$ reagent was characterised using SEM-EDS which showed an even dispersion of silicon throughout the fibre wall (Figure v), with no silica particles evident on the extreme fibre surface, optimal for further silane modification. The location of the silica within the pulp structure was consistent with the results obtained by Donath et al. (Donath et al., 2004), who found that although the pre-hydrolysed silanes gave the greatest weight percent gain, the monomeric silanes resulted in a higher deposition of silica in the cell wall.

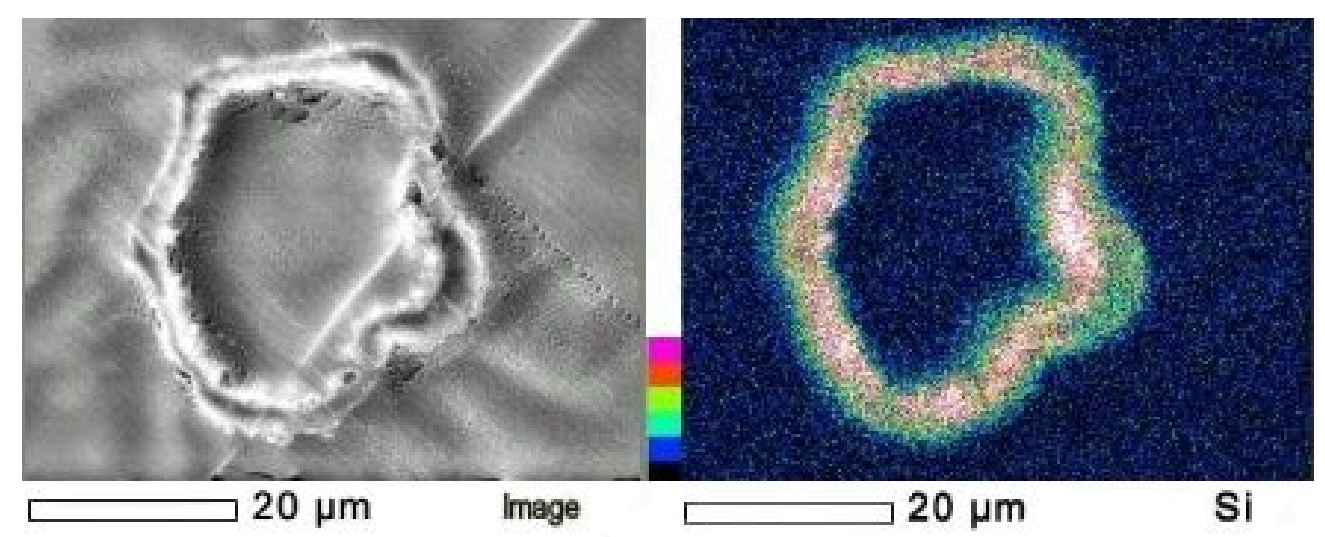

Figure v. SEM and SEM-EDS images of TECS-modified fibre showing silicon distribution in the wall. Lowest concentration of silica is shown in blue false colour, highest in white.

Rinsing the TECS-modified pulp with solvent prior to hydrolysis caused a reduction in the ash content from $20 \%$ to between $14-15 \%$, which suggested that TECS was reacting with the azeotropically-dried pulp directly on addition, prior to hydrolysis. This option gave more control over the destination of the silicon compound which could react with the phenolic groups of the residual lignin and/or with the hydroxyl groups of the cellulose surface. To test this hypothesis, reactions of TECS with guaiacol (as a model for the phenolic components of lignin) and with glucose 1,2-di-O-isopropylidene (as a model for cellulose) were followed by NMR. The isopropylidene derivative was used instead of glucose because of its solubility in the reaction medium, and therefore more ready exchange between the isopropylidene group and the silane- $\mathrm{Cl}$ group to form $\mathrm{HCl}$, isopropanol and the silicon ester. In each case there was evidence for slow reactions taking place, with the $\mathrm{Cl}_{2} \mathrm{Si}(\mathrm{OEt})_{2}$ fraction of the silane reaction mixture disappearing more quickly owing to its higher reactivity. These results showed that direct reactions were feasible, but did not distinguish between the importance of the two components in the wood fibres for anchoring the silane reagent prior to hydrolysis of the ethyl esters to form silica polymer within the fibre wall. 


\subsection{Characterisation of Silica-Modified Kraft Pulp}

The location of the silica within the fibre structure was determined by EDS-SEM (see results above), but did not describe the chemistry of the silica compounds. DRIFT spectra of unbleached Kraft pulp modified with TECS was compared with an unmodified pulp control (Figure vi). The two spectra were nearly identical apart from the presence of a small peak at $1183 \mathrm{~cm}^{-1}$ for the silica modified pulp spectra. Abdelmouleh et al. (Abdelmouleh et al., 2004) assigned this region, and a peak at $1134 \mathrm{~cm}^{-1}$, to the stretching bands of Si-O-Cellulose and Si-O-Si. The TECS-modified unbleached Kraft pulp was also analysed by solid-state ${ }^{29} \mathrm{Si}$ NMR and gave a weak peak at $-108 \mathrm{ppm}$. This spectrum was the same as acid-washed sand, which gave a much stronger peak at -109 ppm, confirming that the silicon in the pulp sample was present as $\mathrm{SiO}_{2}$.

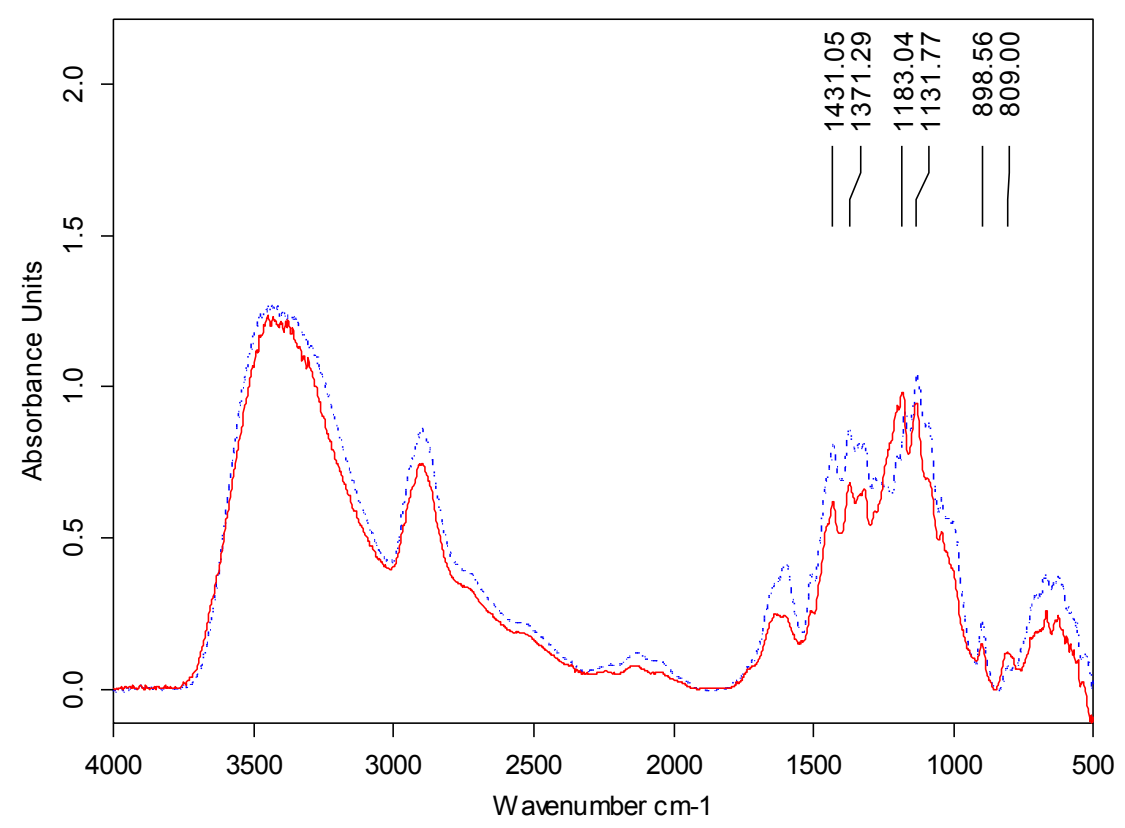

Figure vi: DRIFT spectra of TECS-modified unbleached Kraft pulp (solid line) and unbleached Kraft pulp control (dotted line).

\section{Conclusion}

The objective of selectively filling fibre walls with silica, imparting glass surface properties to woodderived fibre, has been achieved. Azeotropic distillation, allowing concomittant fibre drying and chemical modification, was discovered to be a simple and controllable route to the wall filling process. It was also observed that azeotrope drying alone produced a larger fibre than that obtained from passive air drying, and that the fibre also appeared to be stiffer and more resistant to collapse than the air-dried fibre. 
The novel use of the triethoxychlorosilane reagent has been shown for the first time to be more selective over TEOS or 'Silbond 40' for the formation of silica polymer within the fibre wall, as shown by scanning electron microscopy and EDS. This reagent could find wider application in specifically modifying other hydroxylic polymeric materials.

\section{Acknowledgements}

The financial support from the Foundation for Research, Science and Technology and Chemcolour Industries (NZ) Ltd. is gratefully acknowledged. The authors thank Dr Adya Singh for carrying out the TEM measurements of the silica-modified fibre.

\section{References}

Abdelmouleh, M., Boufi, S., Belgacem, M. N., Duarte, A. P., ben Salah, A., and Gandini, A. Modification of cellulosic fibres with functionalised silanes: development of surface properties. International Journal of Adhesion and Adhesives 24, 43-54. 2004.

R.Askeland, The Science and Engineering of Materials, Chapman and Hall: 1996.

Beshay, A. D., Kokta, B. V., and Daneault, C. Use of wood fibers in thermoplastic composites II: Polyethylene. Polymer Composites 6[4], 261-270. 1985.

Chernyshev, E. A., Lebedev, E. N., Kleshchevnikova, S. I., Komalenkova, N. G., Bykovchenko, V. G., and Tagachenkov, A. A. Study of the reaction of tetraethoxysilane with silicon tetrachloride. Synthesis of triethoxychlorosilane. Zhurnal Obshchei Khimii 65[7], 1142-1144. 1995.

Donath, S., Militz, H., and Mai, C. Wood modification with alkoxysilanes. Wood Science and Technology 38, 555-566. 2004.

Kibblewhite, R. P., 1989. Effects of pulp drying and refining on softwood fibre wall structural organisations. In: Fundamentals of Papermaking, Trans. $9^{\text {th }}$ Fund. Res. Symp., Cambridge, UK, Vol. $1: 121-152$.

Kibblewhite, R. P. and Bailey, D. G. Measurement of fibre cross-section dimensions using image processing. Appita Journal 41[4], 297-303. 1988.

Kibblewhite, R. P. and Bawden, A. D. Structural organizations and papermaking qualities of Kraft, soda A-Q, neutral sulfite-AQ, polysulfide, and polysulfide-AQ pulps. Appita 42[4], 275-281. 1989.

Miller N A, Stirling C D and Langford V S M, "Pinus Radiata Fibre/Thermoplastic Composite Materials", Proc. of the Second Pacific Rim Bio-Based Composite Symposium, Vancouver, Canada 1994:47-54.

Mai, C. and Militz, H. Modification of wood with silicon compounds. Inorganic silicon compounds and sol-gel systems - a review. Wood Science and Technology 37, 339-348. 2004.

Matuana, L. M., Balatinecz, J. J., Park, C. B., and Sodhi, R. N. S. X-ray photoelectron spectroscopy study of silane-treated newsprint-fibers. Wood Science and Technology 33, 259-270. 1999. 
Miyafuji, H. and Saka, S. Topochemistry of $\mathrm{SiO}_{2}$ wood-inorganic composites for enhancing waterrepellency. Materials Science Research International 5[4], 270-275. 1999.

Nazhad, M. M. and Paszner, L. Fundamentals of strength loss in recycled paper. Tappi Journal 77[9], 171-179. 1994.

Persson, P. V., Hafren, J., Fogden, A., Daniel, G., and Iversen, T. Silica nanocasts of wood fibers: A study of cell-wall accessibility and structure. Biomacromolecules 5, 1097-1101. 2004.

Pickering, K. L., Abdalla, A., Ji, C., McDonald, A. G., and Franich, R. A. The effect of silane coupling agents on radiata pine fibre for use in thermoplastic matrix composites. Composites Part A: Applied Science and Manufacturing 34[10], 915-926. 2003.

E.P.Plueddemann, Silane Coupling Agent, Plenum Press: New York, 1984.

Qian, J.-M., Wang, J.-P., Qiao, G.-J., and Jin, Z.-H. Preparation of porous SiC ceramic with woodlike microstructure by sol-gel and carbothermal reduction processing. Journal of the European Ceramic Society 24, 3251-3259. 2004.

Raj, R. G., Kokta, B. V., Maldas, D., and Daneault, C. Use of wood fibers in thermoplastics VII. The effect of coupling agents in polyethylene-wood fiber composites. Journal of Applied Polymer Science 37, 1089-1103. 1989.

Saka, S., Sasaki, M., and Tanahashi, M. Wood-inorganic composites prepared by sol-gel processing I. Wood-inorganic composites with porous structure. Mokuzai Gakkaishi 38[11], 1043-1049. 1992.

Saka, S. and Ueno, T. Several $\mathrm{SiO}_{2}$ wood-inorganic composites and their fire-resisting properties. Wood Science and Technology 31, 457-466. 1997.

Schneider, M. H. and Brebner, K. I. Wood-polymer combinations: the chemical modification of wood by alkoxysilane coupling agents. Wood Science and Technology 19, 67-73. 1985.

Shin, Y., Wang, C., Li, X. S., and Exarhos, G. J. Synthesis of supported carbon nanotubes in mineralized silica-wood composites. Carbon 43[5], 1096-1098. 2005.

Shin, Y., Wang, L. Q., Chang, J. H., Samuels, W. D., and Exarhos, G. J. Morphology control of hierarchically ordered ceramic materials prepared by surfactant-directed sol-gel mineralization of wood cellular structures. Studies in Surface Science and Catalysis 146 (Nanotechnology in mesostructured materials), 447-451. 2003.

E.Sjostrom, Wood Chemistry; fundamentals and applications, Academic Press, Inc.: Orlando, Florida, 1981.

Stone, J. E. and Scallan, A. M. A structural model for the cell wall of water-swollen wood pulp fibres

Tshabalala, M. A. and Gangstad, J. E. Accelerated weathering of wood surfaces coated with multifunctional alkoxysilanes by sol-gel deposition. Journal of Coatings Technology 75[943], 37-43. 2003.

Valadez-Gonzalez, A., Cervantes-Uc, J. M., Olayo, R., and Herrera-Franco, P. J. Chemical modification of henequen fibers with an organosilane coupling agent. Composites: Part B 30, 321-331. 1999.

Weise, U. and Paulapuro, H., 1995. Changes of fiber dimensions during drying. 1995 Internat. Paper Physics Conf. (CPPA and TAPPI):121-124. 
Wood, T. M. and Bhat, G. R. Quantifications of cellulase activity. Methods Enzym. 160, 87-116. 1988.

Woodhams, R. T., Thomas, G., and Rodgers, D. K. Wood fibers as reinforcing fillers for polyolefins. Polymer Engineering and Science 24[15], 1166-1171. 1984.

Xanthos, M. Processing conditions and coupling agent effects on polypropylene/wood flour composites. Plastics and Rubber Processing and Applications 3, 223-228. 1983. 